\title{
Headache Literacy- A definition and theory to help improve patient outcomes of diverse populations and ameliorate headache and headache care disparities
}

Larry Charleston IV, MD ${ }^{1}$

Michele Heisler, MD, MPA ${ }^{2,3}$

1. Department of Neurology, University Of Michigan, Ann Arbor, MI

2. Department of Internal Medicine and Health Behavior and Health Education, University of Michigan, Ann Arbor, MI

3. Center for Clinical Management Research, Ann Arbor Veterans Affairs Health System, Ann Arbor, MI

Corresponding Author:

Larry Charleston IV, MD, FAHS, University of Michigan, Headache Medicine, Assistant Professor, Department of Neurology, PH: 734-936-7910, FAX: 734936-8763, 1914 Taubman Center, 1500 E. Medical Center Dr. SPC 5316, Ann Arbor, Michigan 48109-5316 larrycha@med.umich.edu

Study funding: none

Key words: headache literacy, headache care disparities, headache education, headache disparities, patient outcomes, health disparities

Acknowledgements: We thank Daniel J. Clauw, MD and David A. Williams, PhD for reviewing earlier drafts of this paper.

\section{Disclosures}

Dr. Charleston: no disclosures.

Dr. Heisler: is funded by Grant Number P30DK092926 from the National Institute of Diabetes and Digestive and Kidney Diseases and has no other disclosures to report.

This is the author manuscript accepted for publication and has undergone full peer review but has not been through the copyediting, typesetting, pagination and proofreading process, which may lead to differences between this version and the Version record. Please cite this article as doi:10.1111/ head.12954. 
In this work, we will define headache literacy $(\mathrm{HL})$, provide a rationale for the need for increased HL, discuss what we know and the potential shortcomings of current headache education interventions, and briefly propose how domains of $H L$ can be leveraged to develop interventions to improve headache outcomes and improve disparities in these outcomes.

In spite of growing public and professional awareness, disparities in pain care still persist.(1) The AHRQ reported in 2012 that "disparities in quality and outcomes by income, race, and ethnicity are large and persistent, and were not...improving substantially." (2) At about this same time, an Institute of Medicine (IOM) report on chronic pain identified the development of interventions targeting health disparities in pain as a major research priority and guided the development of the National Pain Strategy (NPS). The National Pain Strategy suggests disparities in pain care including headaches may be due to conscious and unconscious biases and negative attitudes, beliefs, perceptions, misconceptions about higher-risk populations groups (e.g. race and gender biases), lack of sufficient knowledge of behavioral and biological issues that affect pain, management and data to understand pain and its treatment in higher risk and vulnerable populations, and pain itself.(1)

There is a paucity of research regarding racial/ethnic disparities and in methods of ameliorating racial/ethnic disparities in headache medicine. A summary of the literature by Shavers et al suggests racial/ethnic disparities in 
pain may be due to the following: a) limited access to health care and appropriate analgesics; b) patient access to or utilization of pain specialists; c) miscommunication and/or misperceptions about the presence and/or severity of pain; d) patients' attitudes, beliefs, and behaviors that influence the acceptance of appropriate analgesics and analgesic doses; and e) providers' attitudes. knowledge and beliefs about patient pain.(3)

Based on evidence on the etiology of racial/ethnic disparities in pain, we might categorize the sources of disparities in pain as system/environmentgenerated, provider-generated, and/or patient-generated. Headache is the leading pain syndrome and reason for medical consultation, especially for neurological evaluation. $(4,5)$ Tension type headache and migraine are ranked as the second and third most common prevalent disorders, respectively, and migraine is the seventh-highest specific cause of disability worldwide.(6) Disparities in headache and migraine exist in the U.S. (7-13) I hypothesize that a key factor underpinning headache care disparities may lie in patients' headache literacy $(\mathrm{HL})$.

In general, health literacy skills can be defined as 'the personal characteristics and social resources needed for individuals and communities to access, understand, appraise and use information and services to make decisions about health' (World Health Organization, 2015). Health literacy is becoming widely acknowledged to be a significant cause of health disparities. (14-17) Prior literature has not defined or well-described health literacy specifically regarding 
headaches. However, adapting the WHO 2015 definition, I propose a definition of headache literacy $(\mathrm{HL})$ as "the personal characteristics and social resources for individuals and communities to be empowered to access, understand, and use information and services to make informed decisions regarding headaches." Headache literacy may lead to awareness and use of headache-specific treatments, headache specialists, improved communications, decreased misperceptions, decreased medication overuse headache, and early and safe health intervention-seeking behaviors, thereby, reducing morbidity of headache disorders and increasing quality of life.

Rothrock et al demonstrated that patients with intensive education on headaches and appropriate care had a significantly greater reduction in mean MIDAS score, a reduction in mean headache days per month and a greater reduction in functionally incapacitating headache days per month, exhibited less analgesic overuse and need for abortive therapy, were more adherent to prophylactic therapy prescribed, and made fewer headache-related calls to the clinic or unscheduled visits. (18) These researchers then sought to expand their "headache school" program interregionally; however, this program failed for several reasons, many of which appear to be related to methodological issues and not the education itself.(19) Extrapolating from this program, one may consider other methodologies to increase participation and get a better understanding of patient demographics, cultural behaviors and influences, vernacular, etc. (e.g., qualitative, mixed-method, community based participatory 
research). Indeed the authors concluded, "...we learned the bittersweet lesson that such educational initiatives should be tailored to the particular characteristics of the patient population for which they are intended." In addition, studies of headache educational programs would likely need to be well funded and given adequate time to complete and to assess longer-term outcomes after completion of the program. (19)

(1)

On the other hand, although headache literacy has an educational component, it is not just headache knowledge. Attitudes, beliefs, culture and perceptions all play a role in "personal characteristics", an integral part of HL. Social resources contribute to an adequate definition of HL. In addition, effective patient-provider communication is an integral part of HL. The ability to effectively receive and transmit information is essential to $\mathrm{HL}$. In a review of 21 studies, Steward et al found that effective patient-physician communication positively affected patients' outcomes in areas such as pain, anxiety, functional status, and physiologic measures of blood pressure and blood glucose. (20) Interpersonal skills and communication have also been an Accreditation Council for Graduate Medical Education (ACGME) requirement. (21) Patient-provider communication may further improve quality of care and patient satisfaction which may result in better outcomes from headache sufferers.(22)

Instruments such as the short test of functional health literacy in adults (STOFHLA), the rapid adult estimate of adult literacy in medicine (REALM), and new vital sign (NVS) are commonly used to assess health literacy.(23-25) However, these generic measures of health literacy obviously lack many terms 
germane to headache medicine and do not exhaustively take into account "patient characteristics" and/or "social resources" aside from education. Headache literacy requires patients to not only "know" information about headaches, but they also need to be able to understand, access, and utilize the knowledge.

An adequate and comprehensive definition of the key components of headache literacy is an essential first step for the development and evaluation of effective interventions to improve headache literacy and outcomes. Key components likely include headache knowledge, perceptions, beliefs, overall literacy, and other factors influencing headache decision-making (e.g. behaviors, impact, burden, modes of communication, methods of obtaining information, etc.) in patients that may contribute to headache literacy. These elements likely vary among and possibly within different communities, cultures and/or race/ethnic backgrounds, Enhancing HL among individuals may be a critically important mechanism to improve patient outcomes of diverse populations and ameliorate headache/headache care disparities. Well-designed studies are needed to test this hypothesis of the key role of headache literacy in disparities in headache care and outcomes. These in turn will provide the foundation for the development and rigorous evaluation of health system and community-specific interventions to improve headache outcomes. 


\section{References:}

1. Committee. IPRC. National Pain Strategy: A Comprehensive Population Health-Level Strategy for Pain. 2015 Open draft for comment April 2, 20152015 [Available from: http://iprcc.nih.gov/docs/DraftHHSNationalPainStrategy.pdf 2. Services; UDoHaH. 2014 National Healthcare Quality and Disparities Report. May 2015 [ AHRQ Publication No. 15-0007.]. Available from: http://www.ahrq.gov/research/findings/nhqrdr/index.html.

3. Shavers VL, Bakos A, Sheppard VB. Race, ethnicity, and pain among the U.S. adult population. J Health Care Poor Underserved. 2010;21(1):177-220.

4. Pascual J, Combarros O, Leno C, Polo JM, Rebollo M, Berciano J. [Distribution of headache by diagnosis as the reason for neurologic consultation]. Med Clin (Barc). 1995;104(5):161-4.

5. Stewart WF, Roy J, Lipton RB. Migraine prevalence, socioeconomic status, and social causation. Neurology. 2013;81(11):948-55.

6. Vos T, Flaxman AD, Naghavi M, Lozano R, Michaud C, Ezzati M, et al. Years lived with disability (YLDs) for 1160 sequelae of 289 diseases and injuries 19902010: a systematic analysis for the Global Burden of Disease Study 2010. Lancet. 2012;380(9859):2163-96.

7. Heckman BD, Holroyd KA, O'Donnell FJ, Tietjen G, Utley C, Stillman M, et al. Race differences in adherence to headache treatment appointments in persons with headache disorders. J Natl Med Assoc. 2008;100(2):247-55.

8. Heckman BD, Holroyd KA, Tietjen G, O'Donnell FJ, Himawan L, Utley C, et al. Whites and African-Americans in headache specialty clinics respond equally well to treatment. Cephalalgia. 2009;29(6):650-61.

9. Latinovic R, Gulliford M, Ridsdale L. Headache and migraine in primary care: consultation, prescription, and referral rates in a large population. J Neurol Neurosurg Psychiatry. 2006;77(3):385-7.

10. Loder S, Sheikh HU, Loder E. The prevalence, burden, and treatment of severe, frequent, and migraine headaches in US minority populations: statistics from National Survey studies. Headache. 2015;55(2):214-28.

11. Nicholson RA, Rooney M, Vo K, O'Laughlin E, Gordon M. Migraine care among different ethnicities: do disparities exist? Headache. 2006;46(5):754-65.

12. Silberstein S, Loder E, Diamond S, Reed ML, Bigal ME, Lipton RB, et al. Probable migraine in the United States: results of the American Migraine Prevalence and Prevention (AMPP) study. Cephalalgia. 2007;27(3):220-9.

13. Wheeler SD, Carrazana EJ. Delayed diagnosis of cluster headache in AfricanAmerican women. J Natl Med Assoc. 2001;93(1):31-6.

14. Berkman ND, Sheridan SL, Donahue KE, Halpern DJ, Crotty K. Low health literacy and health outcomes: an updated systematic review. Ann Intern Med. 2011;155(2):97-107.

15. Bodie GD, Dutta MJ. Understanding health literacy for strategic health marketing: eHealth literacy, health disparities, and the digital divide. Health Mark Q. 2008;25(1-2):175-203. 
16. Kiechle ES, Bailey SC, Hedlund LA, Viera AJ, Sheridan SL. Different Measures, Different Outcomes? A Systematic Review of Performance-Based versus SelfReported Measures of Health Literacy and Numeracy. J Gen Intern Med. 2015;30(10):1538-46.

17. Rowlands G, Shaw A, Jaswal S, Smith S, Harpham T. Health literacy and the social determinants of health: a qualitative model from adult learners. Health Promot Int. 2015.

18. Rothrock JF, Parada VA, Sims C, Key K, Walters NS, Zweifler RM. The impact of intensive patient education on clinical outcome in a clinic-based migraine population. Headache. 2006;46(5):726-31.

19. Andress-Rothrock D, Rothrock JF. The University of Alabama Interregional Headache School Study: anatomy of a failure. Headache. 2012;52(4):694-7. 20. Stewart M, Brown JB, Boon H, Galajda J, Meredith L, Sangster M. Evidence on patient-doctor communication. Cancer Prev Control. 1999;3(1):25-30.

21. ACGME. Accreditation Council for Graduate Medical Education (ACGME)

Program Requirements for Graduate Medical Education in Neurology 2013

[Available from:

http://www.acgme.org/acgmeweb/Portals/0/PFAssets/ProgramRequirements/18 $\underline{0 \text { neurology 07012014.pdf. }}$

22. Buse DC, Lipton RB. Facilitating communication with patients for improved migraine outcomes. Curr Pain Headache Rep. 2008;12(3):230-6.

23. Gazmararian JA, Baker DW, Williams MV, Parker RM, Scott TL, Green DC, et al. Health literacy among Medicare enrollees in a managed care organization. JAMA. 1999;281(6):545-51.

24. Estrada CA, Martin-Hryniewicz M, Peek BT, Collins C, Byrd JC. Literacy and numeracy skills and anticoagulation control. Am J Med Sci. 2004;328(2):88-93.

25. Weiss BD, Mays MZ, Martz W, Castro KM, DeWalt DA, Pignone MP, et al. Quick assessment of literacy in primary care: the newest vital sign. Ann Fam Med. 2005;3(6):514-22. 


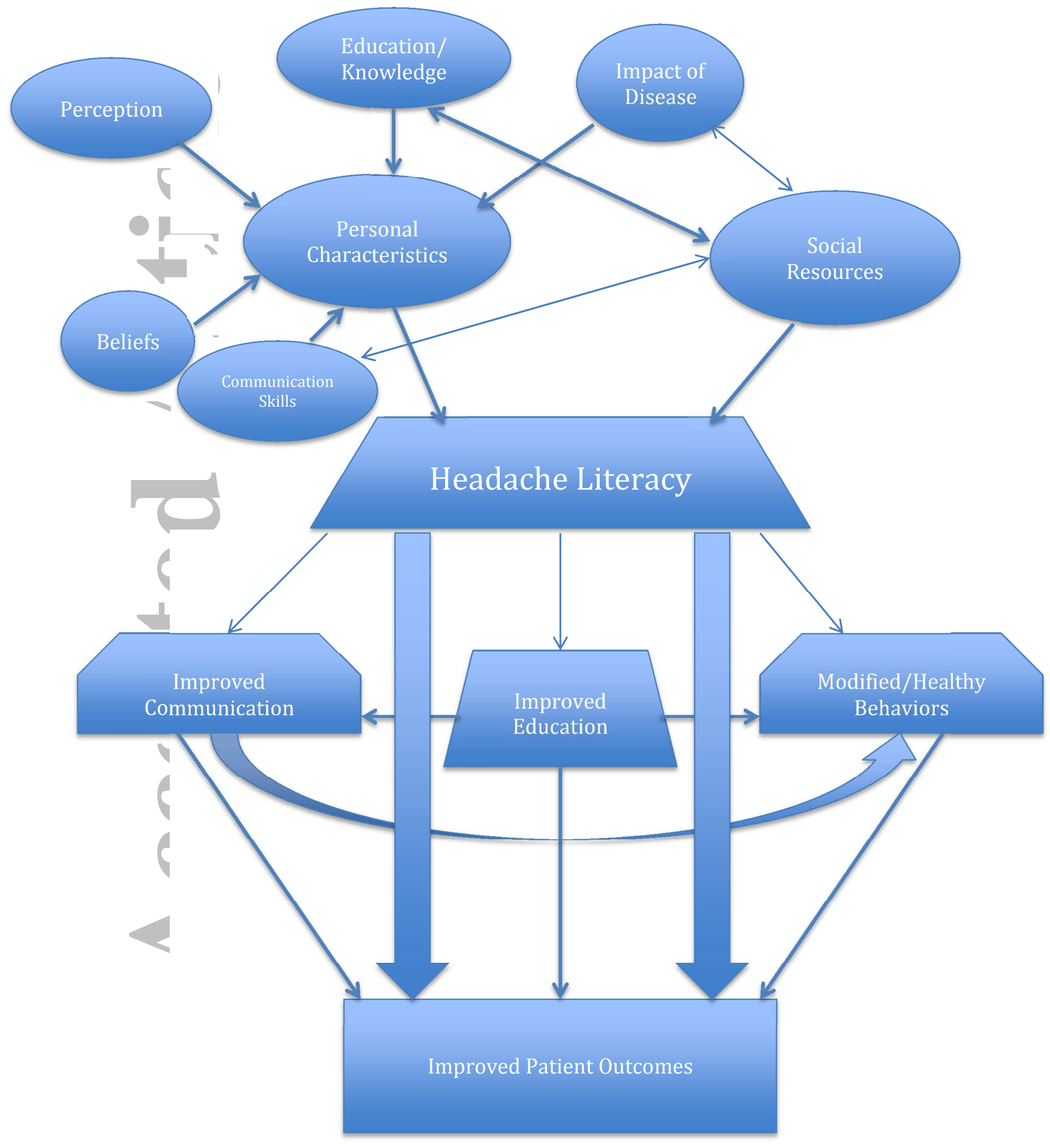

Headache Literacy- A Theoretical Model and Pathway to Improved Patient Outcomes 